\title{
Comparing Simulation Model for Objective QoE Video Evaluation with real IPTV Test Scenario During Appearance of Packet Losses
}

\author{
Nermin Goran, Member, IEEE, Alen Begović, and Namir Škaljo
}

\begin{abstract}
The aim of this article is to explain benefits of the simulation model for objective QoE (Quality of Experience) video evaluation in order to find similarities with real IPTV (Internet Protocol TeleVision) system distribution in a DSL (Digital Subscriber Line) network. In DSL networks, it is known that packet losses appear suddenly and might have a "burst" character, at any time during IPTV service delivery. In addition, these packet losses usually appear in groups and lead to huge degradation of the IPTV video service, which decreases a customer's QoE guaranteed level. Hence, estimation of this degradation in an access network is crucial and this paper explains a simulation model based on SSIM (Structural Similarity Index) analysis, which can be used as one perceptive video quality assessment by imitating a real environment with packet losses. To check this, we compared simulation model cases with the real ones in IPTV video distributed over DSL and exposed to different packet loss appearances.
\end{abstract}

Keywords - IPTV, packet loss QoS/QoE simulation; SSIM, QoE assessment.

\section{INTRODUCTION}

$\mathrm{I}_{\mathrm{c}}^{\mathrm{N}}$ order to check subscriber's subjective reactions to external stimuli, a paradigm of evaluation of Quality of Experience (QoE) is introduced in [1]. According to ITU (International Telecommunication Union) organisation, the QoE video evaluation refers to the common acceptance of video service subjectively perceived by the end user [2]. Subjective QoE estimation is related to a testing process conducted by a group of participants. The most common measure for subjective testing is MOS (Mean Opinion Score) [3],[4]. Nevertheless, development of video applications in ICT industry and their delivery generate

Paper received March 27, 2020; revised November 15, 2020; accepted November 18, 2020. Date of publication December 25, 2020. The associate editor coordinating the review of this manuscript and approving it for publication was Prof. Grozdan Petrović.

This paper is revised and expanded version of the paper presented at the 27th Telecommunications Forum TELFOR 2019 [30].

Nermin Goran is with Faculty of Transport and Communications, University of Sarajevo, Zmaja od Bosne bb, 71000 Sarajevo, Bosnia and Herzegovina, and BH Telecom JSC Sarajevo (phone: +38730509801; e-mail: nermin.goran@bhtelecom.ba).

Alen Begović is with Faculty of Electrical Engineering, University of Sarajevo, Zmaja od Bosne bb, 71000 Sarajevo, and BH Telecom JSC Sarajevo, Franca Lehara 7, 71000 Sarajevo, Bosnia and Herzegovina (phone: +38732442222; e-mail: alen.begovic@bhtelecom.ba).

Namir Škaljo is with University of Zenica, Polytechnic Faculty, Fakultetska bb, 72000 Zenica, Bosnia and Herzegovina (phone: +38732242000; e-mail: namir.skaljo@bhtelecom.ba). many of E2EQoE (End-to-End QoE) objective methods for estimating video service [5]. Authors in [6] emphasised the importance of objective testing in relation to a quantitative subjective approach. Classifications, definitions and a comparison of performances of different objective methods of video service estimation are given in [7]. In addition, the authors in [8] presented important steps towards understanding the new methods and different video metrics. They have used benchmarking that consists of perceptual metrics for video quality testing in order to compare different techniques of video processing. They have also noticed that there is a need for expanding the methods of objective video quality testing and use of so-called FR (Full Reference), RR (Reduced Reference) as well as NR (No Reference) methods. On the other hand, most authors used PSNR (Peak Signal-to-Noise Ratio) as video metric for objective video signal testing. Although, along with PSNR other authors very commonly used SSIM (Structural Similarity Index), PEVQ (Perceptual Evaluation of Video Quality) and VQM (Video Quality Metric) [9-12]. Along with QoE analyses, there are different models and approaches to QoS (Quality of Service) issues in telecommunication networks. The definition of so-called perceived QoS, given by ITU standardisation body in recommendation E.800, is ability of a network or a part of the network to assure the functions referring to communication among the users. In addition, the explanation about perceived QoS is given in ITU recommendation G.1080 [13]. On the contrary, ITU recommendation P.910 [14] defines QoE as a user's decision to continue using the service depending on the quality, the price and the response to complaints and problems of users by the service suppliers and operators. At the same time, there are many models, which explain relationship between QoS and QoE. Packet loss is only one of QoS indicators that can be related with a low QoE level. The authors in [8] and [15-18] conducted the analysis of distorted QoS indicators during video service delivery. They noticed a strong correlation between packet loss appearance and QoE value. Because of this, there are many implemented online monitoring systems of real-time QoE considering packet losses [19]-[21]. However, it is a very common case that there is no monitoring system in access network. In that case, QoE evaluation can be only achieved by contacting customers, which is unpopular and difficult. On the other hand, the simulation described in this paper analyses all possible cases of packet losses that we can use for QoE evaluation in different network conditions. QoE 
testing of IPTV service under different network conditions and its concept are shown in [22]-[25].

In addition, video service delivery in real time has been tested in [26] and [27]. Simulations of video service testing models are presented in [28] and [29]. This paper represents an extended version of paper published on conference TELFOR 2019. Accordingly, the paper analyses a simulation model that imitates video service transmission via a simulation network in which packet losses suddenly appear. It is very close to a real IPTV scenario where packet loss can appear at every moment over the QoS (Quality of Service) unreliable access network with an unstable line rate such as DSL. For the sake of testing, we made a lab in BH Telecom JSC Sarajevo and we used IPTV video with a specific GoP (Group of Pictures) structure (I frame, P, frame, $16 \mathrm{~B}$ frames) which was the same as the structure in a simulation network. At the end of this simulation network we recorded transmitted video and compared it with the original one by SSIM method. At the same time, in the real IPTV network, we generated the same video with the same GoP structure and the same packet loss scenarios. In order to get packet losses in the real IPTV network we used an external disturbance generator which was introduced in DSL line. These events lead to different video degradations and afflict different I, P or B video frames which led to different SSIM values as in simulation cases. The following chapters deal with the necessary steps to perform the testing, simulation cases and results. Mathematical relations that describe the analytical method of the same process are given in [31]. The conclusion is given at the end of the paper.

\section{Description OF USING The Simulation For QoE TESTING IN REAL NETWORK}

The procedure of simulation method for QoE testing in a real network will be explained in this chapter. Considering Fig. 1. a), we can notice one simplified IPTV system that represents a usual scheme of transfer video service over a telecommunication infrastructure of a telecom operator.

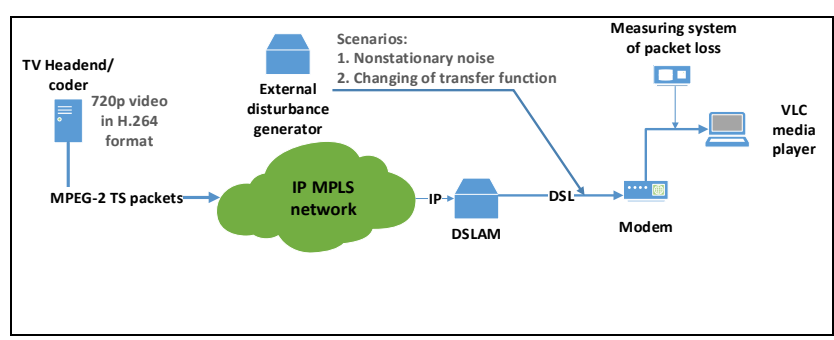

a)

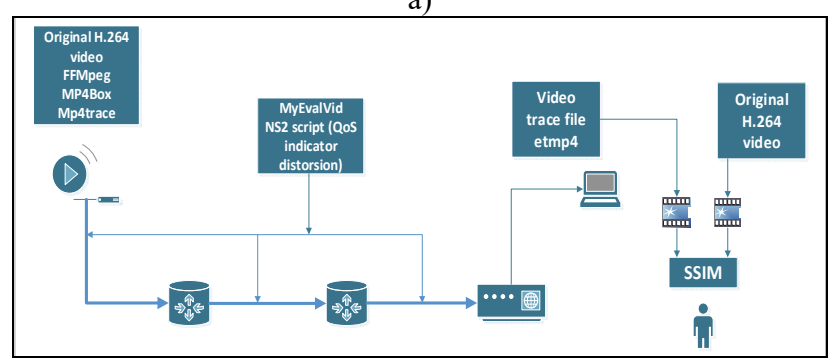

b)

Fig. 1. Simplified scheme of a) real IPTV system b) simulation model.
We monitored a telecommunication network and concluded that the telecommunication network was QoS prone to DSLAM (Digital Subscriber Line Access Multiplexer), precisely to the system of copper twisted pairs. But in this part of telecommunication network packet losses can happen accidentally and then they often have explosive character. Their appearance can be related to the appearance of nonstationary noise and/or unpredictable changes of crosstalk transfer function of twisted pair system. In our lab we artificially created those disturbances with an external disturbance generator which was installed between DSLAM and the user and we got different cases of packet losses and different video degradations for a few scenarios.

To find similarities and the possibility of using simulation for these scenarios, we created the model shown in Fig. 1. b).

The simulation model, depicted in Fig. 1. b), shows one method that can be useful for evaluation of QoE (objective and technical) without user involvement in evaluation process. To check QoE (in this case perceptive Quality of Service) in the simulation model we have used a few of the tools available for academic research, such as NS2 simulator expanded with EvalVid part and VQMT (Video Quality Measurement Tool) that can be used for an objective evaluation of QoE.

The main purpose of using this model is to create opportunities for the evaluation of DSL lines without technicians going out into the field to measure degradation.

In addition, we have created this model to simulate the real conditions that may arise during the transmission of IPTV services. In a real video processing stage (Fig. 1a), we used FFmpeg tool after the video content was recorded on VLC in H.264 format for two separate video sequences (two twisted pair lines one with the original and the other with a disturbed signal). Then, the original video was compared to the FR method with the degraded video that was under the influence of systematic packet loss caused by an external generator. FR method as an objective method was used because degraded and original video appear on the testing side. To obtain SSIM values we used VQMT tool. SSIM values obtained by FR method were used to objectively evaluate the video service quality due to a better correlation with MOS than PSNR according to [9]. This method implies video quality testing by comparing the original and degraded video sequence with all of its similarities [7]. We monitored packet losses for different scenarios on a measuring system (Fig. 1a) which was integrated in IPTV delivery system. After the experimental testing, the original video was introduced again, according to Fig. 1b, into NS2 simulator. At this point, video transmission was simulated and the effects of packet loss were created similar to the ones on a degraded line in real environment [31].

To check the similarities of simulated and experimental cases we used three scenarios during the testing. The first one refers to the incidental appearance of high packet losses, the second case refers to the appearance of packet losses of the similar intensity with a certain degree of repetition in different time intervals, and the third one refers to the appearance of low block packet losses. In NS2 
simulator, a simplified telecommunication network structure was formed for imitating the real conditions and assumptions. The original IPTV video was previously prepared for the distribution via a simulated network with the use of the following tools: FFmpeg, Mp4Box and Mp4trace. In order to create video degradations in the simulated environment, QoS distortions in the form of packet losses were introduced between the end node and the end switch. The number of packet losses in the simulated case was derived from the measured values of the losses in real IPTV traffic. After the simulation, the tested video, as in the real case, was compared to the original one by VQMT tool to obtain SSIM values. There are the steps and code of simulation method processes in details in [31]-[32].

\section{Analysis of Simulation in CASE of the APPEARANCE OF HIGH PACKET LOSSES}

The first scenario should confirm the efficiency of the simulation method for evaluation of SSIM values in the case of a single block packet loss appearance of a long duration.

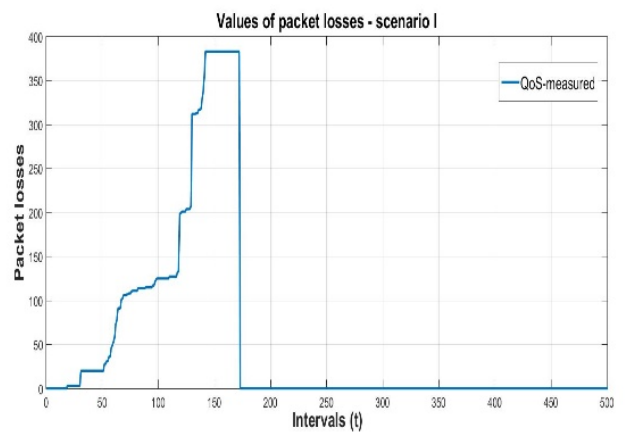

Fig. 2. Measured values of packet losses - I case.

At first, we generated packet losses in a real network by an external disturbance generator and followed the results in a measuring system. Fig. 2 depicts that case i.e. the measured values of packet loss in a real network. Obviously, packet loss and count of lost packets can be noticed in a time duration of $200 \mathrm{~ms}$. The same number of lost packets was introduced into the simulation model. During simulation we used the same video as in a real network and after that a post-process analysis was done (by FFmpeg and VQMT). We obtained SSIM values for all video frames in two networks, the simulated one and in the real network. A comparison of degraded and original video of both networks is shown in Fig. 3.

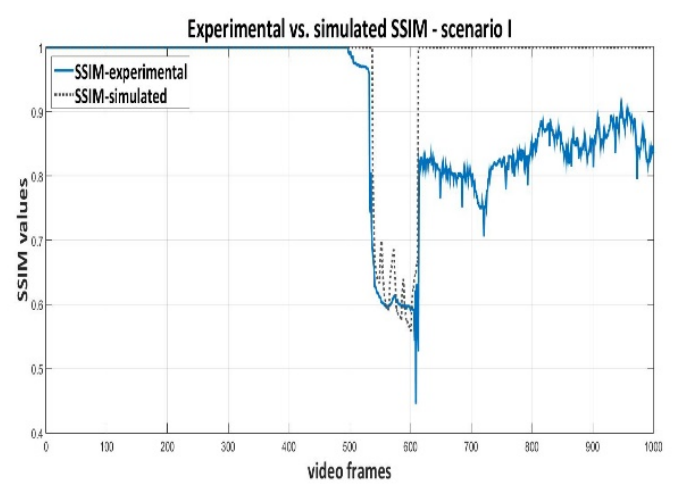

Fig. 3. SSIM values - I case.

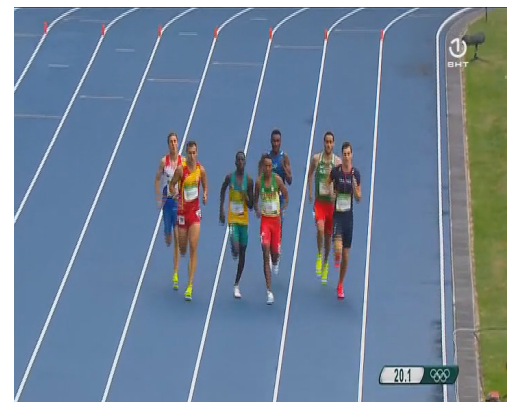

a)

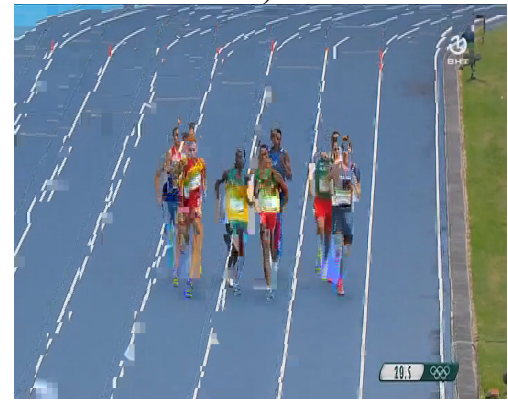

b)

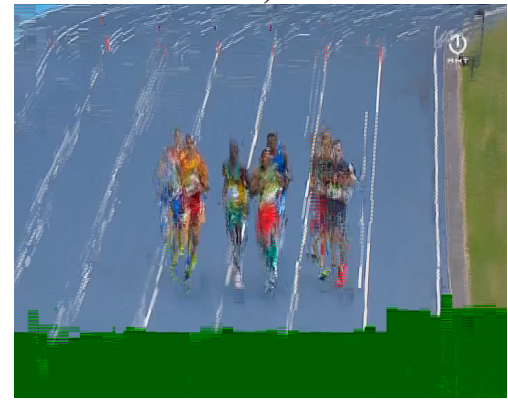

c)

Fig. 4. First degraded video frame at the beginning of appearance of packet losses - I case, a) Original video frame, b) Simulated case, c) Real case.

Obviously, two SSIM graphs, one created by real video testing and the other one formed by the simulation, have contours of a very similar form. In this case, it is clear that objectively perceived QoS estimation via SSIM (that can be mapped to objective QoE) is very similar to the estimation given by the simulation model. Furthermore, SSIM RMSE equals 0.08, while the correlation among SSIM values in simulated and real case equals 0.82 . Certain differences noticeable in the graphs (Fig. 3) refer to somewhat worse values lasting longer in the real network than in the simulated one. We suppose that is the consequence of the underestimation of the fault in the simulation model. Fig. 4. b) depicts degradation of the first video frame at the beginning of appearance of packet losses in simulation channel. During the video reproduction on simulated line, the video was equal to the original one at the beginning of reproduction up to the moment of appearance of the disturbance. The disturbance caused video degradations because of the loss of complete frames or some parts of other frames (in total 168). In Fig. 4. c), it can be noticed that the degradation is visually worse in real than in simulated case even for degradation of the first frame. We suppose that the reason is the GoP structure and appearance of the first I-frame loss during real MPEGTS video format distribution. In addition, during the disturbance, the 
degraded video frames had similar distortions according to SSIM values in simulated case (Fig. 3.). After the disappearance of packet loss, it is important to emphasize that we visually noticed the match between absences of video degradations in both cases in spite of different SSIM graphs (Fig. 3.), which probably indicates the loss of frame synchronism in a real IPTV case.

\section{Analysis of the Model in CASE of Group Block PACKET LOSSES}

The next scenario tests the efficiency of the model described in the paper in order to evaluate SSIM values in the case of appearance of packet losses of the similar intensity with a certain degree of repetition in different time intervals.

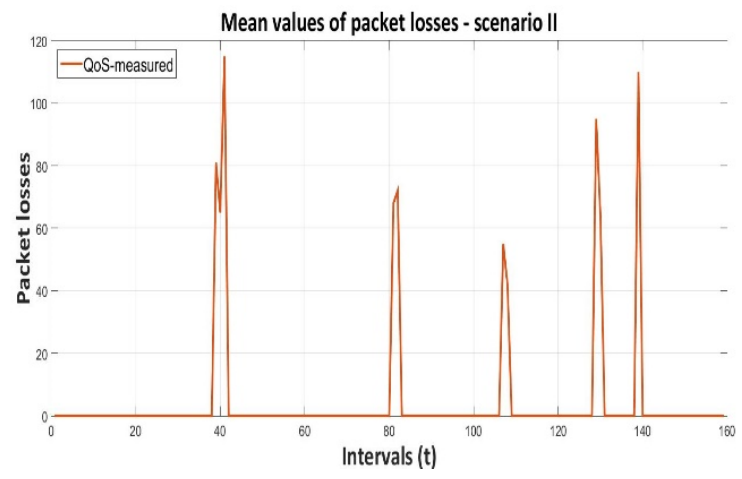

Fig. 5. Measured values of packet losses - II case.

Fig. 5 depicts the values that are measured during video distribution in a real IPTV network. In this case, packet losses of a specific duration appeared in different time intervals. For this purpose, we used a lab where several sequences of the original video were compared to the same number of sequences of the tested video which was degraded by an external generator which caused packet losses in specific time intervals. For simulation purposes as in the previous case, the same packet losses were introduced in NS2 simulator in accordance with the measured values, depicted in Fig. 5.

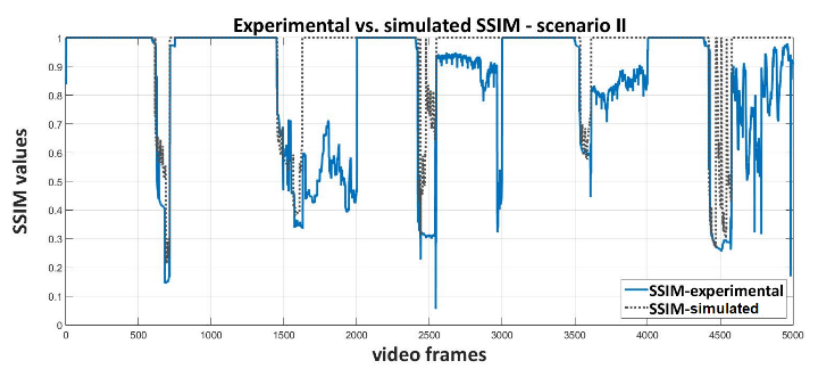

Fig. 6. SSIM values - II case.

Furthermore, in chosen time intervals, in both networks, degradations of QoS parameters were producing video disturbances which are recorded and simultaneously compared to the original video with the use of SSIM method. Fig. 6 depicts two graphs, one that shows SSIM values obtained by comparing degraded and original video marked in blue colour, and the other that shows SSIM values obtained by comparing simulation of degraded and original video marked in grey colour. It can be noticed that SSIM values change especially after the appearance and disappearance of video degradation. There is a clear match between the appearance of disturbance and video degradation in both cases, but also irregularity after the disturbance ends.

As an explanation to this matching, Table 1 shows Pearson's correlation coefficients between experimental and simulated SSIM values for each video sequence from Fig.6. SSIM values for all frames between cases in real time and simulated cases are compared and correlations between them are calculated. The table clearly shows that correlation coefficients for different sequences are not equal although the graphs show a similar trend. As in the first tested case, the video had the clear reproduction after the absence of degradations which fully corresponds to the simulation and little less to a real IPTV distribution. In this network, the most probable cause of it is the disposition of GoP, i.e. I, $\mathrm{P}$ and B frames inside MPEGTS transport packets which additionally generates the loss of synchronism among the frames after the degradation. In the simulation case, I, P and B frames are transmitted over RTP (Real Time Protocol), which has a better response to synchronization issues. Obviously, in a real IPTV network a certain amount of time is necessary for synchronisation to be obtained again. If SSIM values were neglected after the end of video degradation, then the correlation coefficient between the two cases would be much higher than those depicted in Table 1.

TABLE 1: CORRELATION AMONG SSIM VALUES OF COMPARING EXPERIMENTAL AND SIMULATED VIDEO SEQUENCES - II CASE.

\begin{tabular}{|l|c|c|c|c|c|}
\hline & I part & II part & III part & IV part & V part \\
\hline Correlation & 0.94 & 0.45 & 0.77 & 0.72 & 0.75 \\
\hline
\end{tabular}

\section{Analysis of the Model in CASE of Low Rate PACKET LOSS DISTRIBUTION}

Also, and in the last case, the measured values of packet losses generated in the lab i.e. in the real IPTV network are given in Fig. 7.

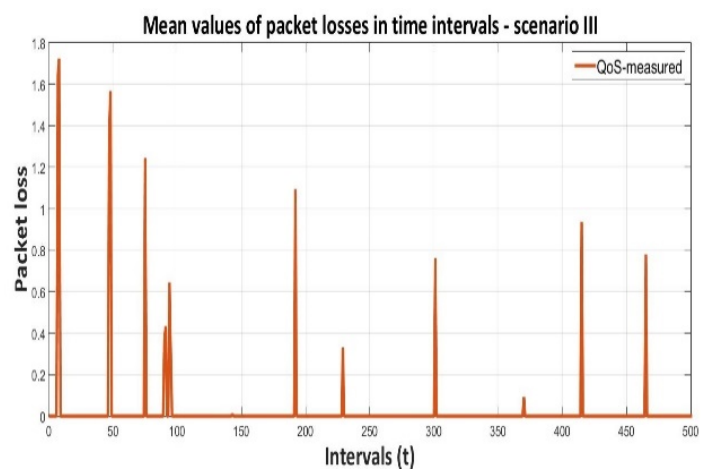

Fig. 7. Measured values of packet losses - III case.

In this case, we generated packet loss more often than in the second case, but at the same time with a small number of loss packets. At the same time, we captured degraded video in order to evaluate SSIM values with original video which was captured at the same time on the line with no packet losses. After introducing these losses into the simulation model, the SSIM method for comparing video frames was carried out again, disturbances were simulated in NS2 and then the simulated video was compared to the 
original video taken in real IPTV environment. SSIM values for both cases are depicted in Fig. 8. and their appearance matches the appearance of temporary disturbances.

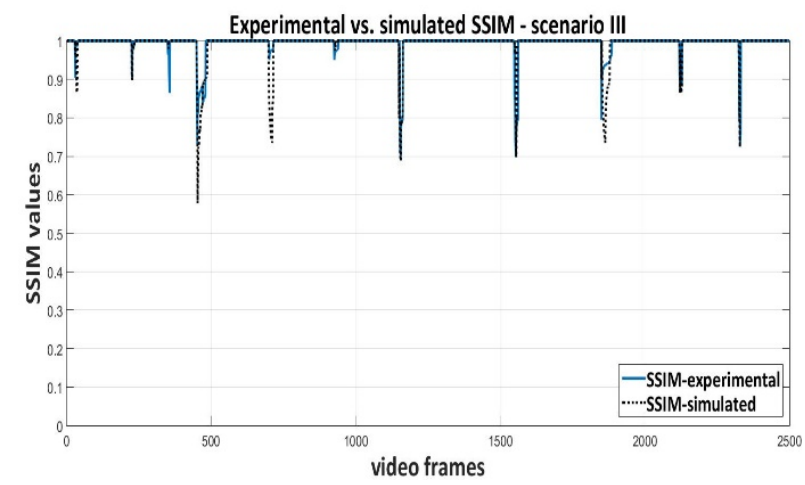

Fig. 8. SSIM values - III case.

The correlation degree between simulated and SSIM values in real environment is very high and varies from 0.89 to 0.99 . Thus, the model evaluates objective QoE very well.



a)

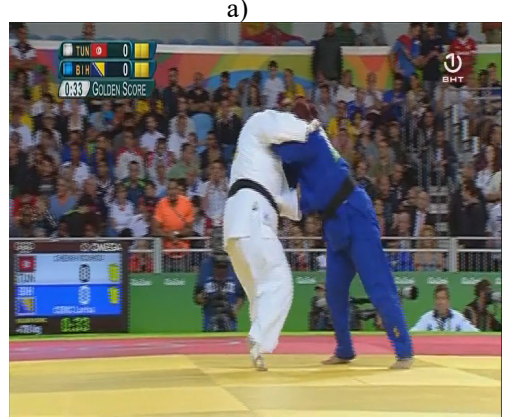

b)

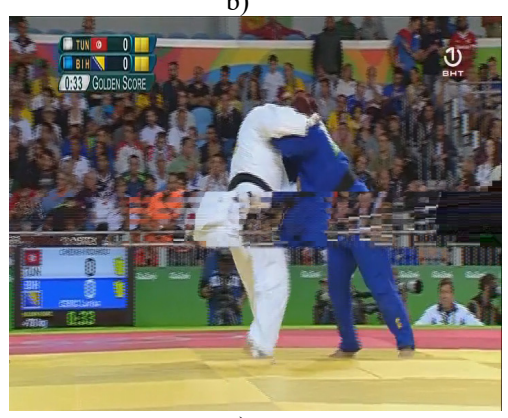

c)

Fig. 9. First degraded video frame at the beginning of appearance of packet losses - III case, a) Original video frame, b) Simulated case, c) Real case.

Fig. 9. depicts the first degraded video frame for both cases i.e. after the appearance of the first disturbance in the communication channel or in the simulator. In this case, as in the previous ones, video degradations were somewhat worse in real environment than in simulation. On the other hand, this case had the best correlation between the results because there were moments where degradations matched in both networks.

\section{CONCLUSIONS}

Analysis of the most common cases gives a clear insight into objective QoE evaluations (QoS perceived quality) and their good visual correlation to video degradations in real and simulated environment. In both cases, the first and the second, when packet losses afflict I frames used for prediction of $\mathrm{P}$ and $\mathrm{B}$ frames, degradation of the real video stretches out to GoP structure. Thus, the disturbances in a real network are more intense. In these cases, the simulation model assumes these degradations well and after their absence, the video is synchronised well again. On the other hand, in real environment this wasn't the case probably due to the problem with synchronism. We suppose this is so because of MPEGTS organization. MPEGTS has proposed structures of transport packets and many of them might carry I frames which leads to tremendous degradations. In the third case, the simulation SSIM values have good matching with SSIM values in real environment.

This can be explained by the possibility of low disturbances which contaminate important I frames. In this case, we suppose that the GoP structure does not influence the appearance of the degradation as in other two cases.

Despite certain deviations in other two cases, the conclusion is that the simulation model is able to predict the moments when the packet losses appear and how long they last, but it underestimates the real effects on QoE to a lesser extent. However, an interesting detail is that the absence of degradation during video observation does not match the testing in a real IPTV network but the simulation model and therefore, this should be a subject of further research.

\section{REFERENCES}

[1] Kjell Brunnström, Sergio Ariel Beker, Katrien De Moor, Ann Dooms, Sebastian Egger "Qualinet White Paper on Definitions of Quality of Experience", 2013.

[2] ITU-T Recommendation, ITU-T SG12, "Definition of Quality of Experience", COM12- LS 62 - E, TD 109rev2 (PLEN/12), Geneva, Jan. 2007.

[3] ITU-T Recommendation, ITU-T P.800.1, "Mean Opinion Score (MOS) Terminology”, 2003.

[4] ITU-T Recommendation, ITU-T P.10/G.100, "Vocabulary for performance and quality of service. Amendment 2: New definitions for inclusion in Recommendation ITU-T P.10/G.100”, Jul 2008.

[5] K. Bouraqia, E. Sabir, M. Sadik and L. Ladid, "Quality of Experience for Streaming Services: Measurements, Challenges and Insights," in IEEE Access, vol. 8, pp. 13341-13361, 2020.

[6] Brooks P.,Hestnes B., "User measures of quality of experience: Why being objective and quantitative is important," in IEEE Network, vol. 24, no. 2, pp. 8-13, Mar.-Apr. 2010.

[7] S. Chikkerur, V. Sundaram, M. Reisslein and L. J. Karam, "Objective Video Quality Assessment Methods: A Classification, Review, and Performance Comparison," in IEEE Transactions on Broadcasting, vol. 57, no. 2, pp. 165-182, June 2011.

[8] Chen, Yanjiao \& Wu, Kaishun \& Zhang, Qian, "From QoS to QoE: A Tutorial on Video Quality Assessment," IEEE Communications Surveys \& Tutorials, IEEE vol 17, pp. 1126-1165, 2015.

[9] Z. Wang, A. Bovik, H. Sheikh, E. Simoncelli, "Image quality assessment: from error visibility to structural similarity," in IEEE Transactions on Image Processing, vol. 13, no. 4, pp. 600-612, Apr. 2004. 
[10] P. Ramos, J. Salmerón, R. Leal, F. Vidal, "Estimating Perceived Video Quality from Objective Parameters in Video over IP Services," in 7th International Conference on Digital Telecommunications (ICDT 2012), Chamonix, France, Apr. 2012, pp. 65-68.

[11] Tianyi Wang, Pervez, A. and Hua Zou, "VQM-based QoS/QoE mapping for streaming video" in Proceedings of the $3 \mathrm{rd}$ IEEE International Conference on Broadband Network \& Multimedia Technology (IC-BNMT), Beijing, China, October 2010, pp. 807-812.

[12] B. Ciubotaru, G. M. Muntean, G. Ghinea, "Objective assessment of region of interest-aware adaptive multimedia streaming quality", in IEEE Transactions on Broadcasting, vol. 55, no. 2, pp. 202-212, Jun 2009.

[13] ITU-T Recommendation, ITU-T G.1080, "Quality of Experience Requirements for IPTV Services", December 2008.

[14] ITU-T Recommendation, ITU-T P.910, "Subjective video quality assessment methods for multimedia applications", 2008.

[15] J. Frnda, J. Nedoma, J. Vanus, R. Martinek, "A Hybrid QoS-QoE Estimation System for IPTV Service," in Electronics, vol 8., Issue 5, pp. 585, 2019.

[16] N. Rao et al., "Analysis of the Effect of QoS on Video Conferencing QoE," 2019 15th International Wireless Communications \& Mobile Computing Conference (IWCMC), Tangier, Morocco, 2019, pp. 1267-1272,

[17] M. Vaser and G. Maggiore, "Improved VoLTE QoE Estimation Procedure using Network Performance Metrics," 2019 Eleventh International Conference on Ubiquitous and Future Networks (ICUFN), Zagreb, Croatia, 2019, pp. 361-365.

[18] N. Goran, B. Bečiragić and M. Hadžialić, “An example of mapping the degradation of network parameters with video QoE parameters in case of IPTV service," 2016 XI International Symposium on Telecommunications (BIHTEL), Sarajevo, 2016, pp. 1-5.

[19] A. Binsahaq, T. R. Sheltami and K. Salah, "A Survey on Autonomic Provisioning and Management of QoS in SDN Networks," in IEEE Access, vol. 7, pp. 73384-73435, 2019.

[20] E. Faghihi and M. Behdadfar, "Adaptive Resource Utilization and Quality Management in VoIP Networks with Quantitative Relationship between QoE and QoS Using HMM," 2020 International Conference on Information Networking (ICOIN), Barcelona, Spain, 2020, pp. 493-498.

[21] A. J. Garcia, M. Toril, P. Oliver, S. Luna-Ramirez and R. Garcia, "Big Data Analytics for Automated QoE Management in Mobile Networks," in IEEE Communications Magazine, vol. 57, no. 8, pp. 91-97, August 2019.
[22] M. N. Zapater and G. Bressan, "A proposed approach for quality of experience assurance for IPTV," in Proceedings of 1st International Conference on the Digital Society (ICDS), Guadeloupe, France, January 2007, pp. 1-25.

[23] D. Hands, O. V. Barriac, and F. Telecom, "Standardization activities in the itu for a QoE assessment of iptv," IEEE Communications Magazine, vol. 46, no. 2, pp. 78-84, 2008.

[24] G. Baltoglou, E. Karapistoli, P. Chatzimisios, "Real-world IPTV network measurements," in Proceedings of the 2011 IEEE Symposium on Computers and Communications (ISCC), Kerkyra, Greece, Jul. 2011, pp. 830-835.

[25] Agrawal, D., Beigi, M. S., Bisdikian, C., Kang-Won Lee, "Planning and managing the IPTV service deployment," in proceedings of 10th IFIP/IEEE International Symposium on Integrated Network Management, Munich, Germany, May 2007, pp. 353-362.

[26] Z. Duanmu, K. Ma and Z. Wang, "Quality-of-Experience for Adaptive Streaming Videos: An Expectation Confirmation Theory Motivated Approach," in IEEE Transactions on Image Processing, vol. 27 , no. 12 , pp. $6135-6146$, Dec. 2018.

[27] P. Juluri, V. Tamarapalli and D. Medhi, "Measurement of Quality of Experience of Video-on-Demand Services: A Survey," in IEEE Communications Surveys \& Tutorials, vol. 18, no. 1, pp. 401-418, First quarter 2016.

[28] J.V.C. Carmona, E.M. Carvalho de Matos, B.S.L. Castro, F.J.B. Barros, M.C.d.A. Neto, E.G. Pelaes, "Video loss prediction model in wireless networks", PLoS ONE vol. 14, no. 3, pp. 1-19, 2019, https://doi.org/10.1371/journal.pone.0212407.

[29] S. Poojary et al., "Analysis of QoE for adaptive video streaming over wireless networks," 2018 16th International Symposium on Modeling and Optimization in Mobile, Ad Hoc, and Wireless Networks (WiOpt), Shanghai, 2018, pp. 1-8.

[30] N. Goran, A. Begović and N. Škaljo, "Comparing simulation model for objective QoE video evaluation with real IPTV test scenario during appearance of packet losses," 2019 27th Telecommunications Forum (TELFOR), Belgrade, Serbia, 2019, pp. 1-4, doi: 10.1109/TELFOR48224.2019.8971318.

[31] N. Goran, M. Hadžialić, "Mathematical Bottom-to-Up Approach in Video Quality Estimation Based on PHY and MAC Parameters," in IEEE Access, IEEE, pp. 25657 - 25670, 2017.

[32] https://github.com/gnermin/ns2simulator

[33] NS2 Learning Guide version 2, Dr. Chih-Heng Ke, Web: http://csie.nqu.edu.tw/smallko/ns2/ns2.htm. 